\title{
Equipment Review
}

\section{Pocket mask ventilation: a critical reappraisal}

Provision of adequate ventilation requires an open secure airway and a means to deliver adequate volumes of air. Oxygen enrichment, although desirable, does not appear to be as critical as adequate volume delivery. The American Heart Association (1974) recommends a minimum of $800 \mathrm{cc}$ tidal volume for optimal treatment of cardiopulmonary arrest.

The pocket mask (Laerdal Medical Corporation) is a compact inexpensive clear polyvinyl device moulded to fit over the nose and mouth and fitted with a portal for oxygen supplementation (Fig. 1). It is collapsible to fit easily into a coat pocket and, therefore, it is portable and accessible. Unlike the bag-valve-mask unit, both hands of the rescuer remain free to achieve a secure fit and eliminate air leak. The use of the rescuer's own lungs for ventilation encourages adequate inspiratory volumes.

In 1980 use of the bag-valve-mask for ventilation was impugned in a comparative study from the Medical College of Virginia (Harrison et al., 1982). Ventilatory volumes delivered to a recording resuscitation manikin were compared using the bag-valve-mask (BVM), endotracheal tube (ETT), esophageal obturator airway (EOA) and a pocket mask (PM). When the rescuer's own lungs were used with the EOA and PM, volumes delivered to the 'lungs' were comparable to ETT veritilation. Volumes delivered by BVM averaged $500 \mathrm{cc}$.

Elling \& Politis (1983) compared the use of the bag-valve-mask and pocket mask and found that more than $50 \%$ of the 320 emergency medical technicians studied were not capable of adequate ventilation using the bag-valve-mask. He recommended that future educational courses teach the bag-valve-mask skill as a four hand-two person exercise. Pocket mask ventilation was determined to be far superior, achieving ventilatory volumes approaching one litre and surpassing all BVM devices tested. In a presentation before the Society of Critical Care Medicine in June 1982, Jesudian et al. confirmed Elling's findings and demonstrated that the mean delivered volume by the BVM single rescuer technique was $592 \pm 78 \mathrm{ml}$ whereas it was $1132 \pm 53 \mathrm{ml}$ when two rescuers were participating.

It is clear from these recent reports that the pocket mask is a superior form of ventilation and reliably delivers a litre or more of ventilatory volume with a single rescuer using the rescuer's own lungs. Clinical data correlating method and volume of ventilation with the level of patient oxygenation, however, is currently unavailable. This data is essential to confirm what advantage, if any, the pocket mask may have over other forms of mechanical ventilation.

\section{SUMMARY}

The pocket mask is a convenient and cost effective method of ventilation when used 


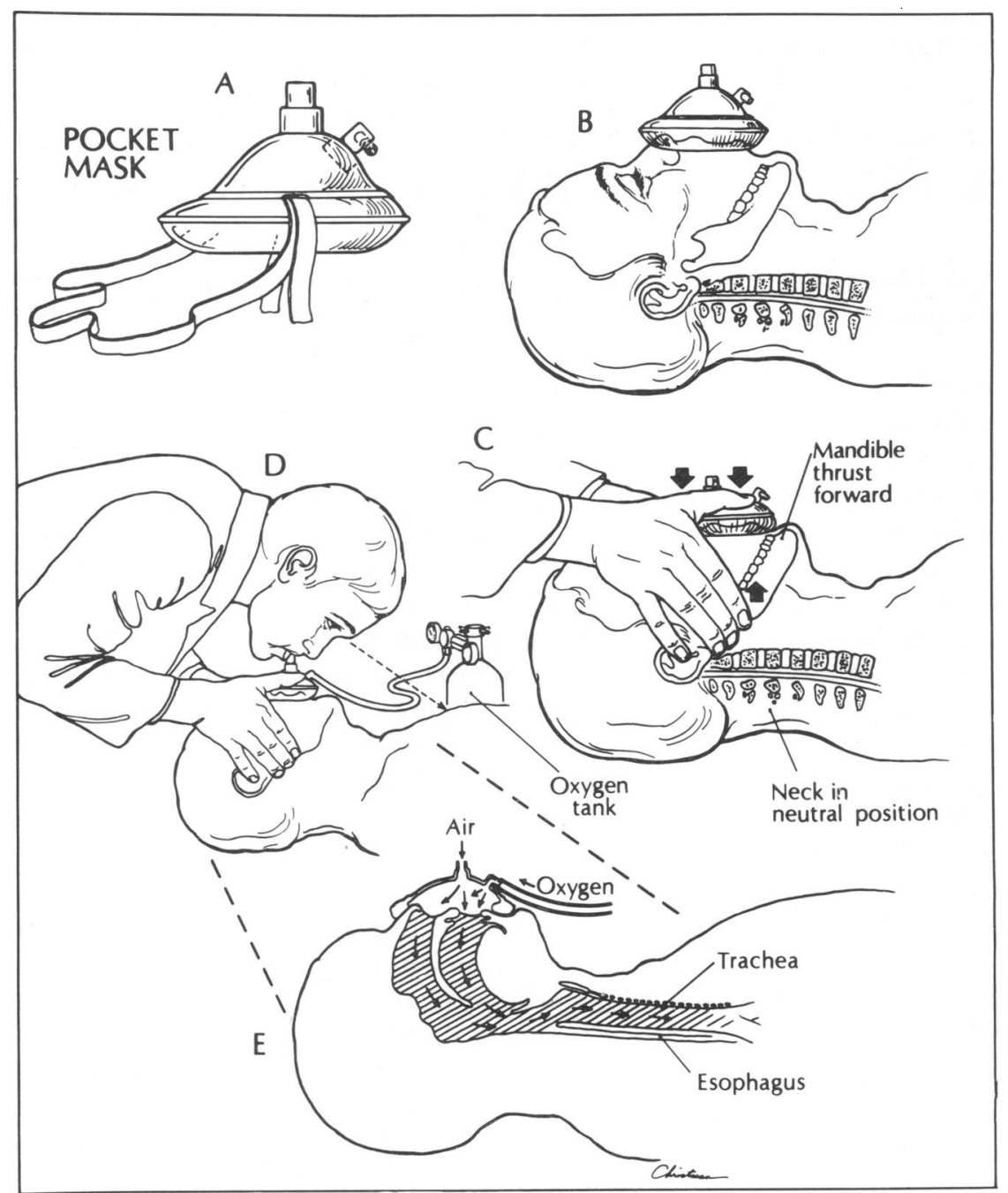

Fig. 1 Pocket mask ventilation demonstrating maintenance of open airway and method of ventilation with oxygen enrichment.

with the rescuer's lungs and appears to offer a clear advantage in terms of volume delivered during the early treatment of respiratory arrest. Clinical data is needed tof confirm the advantage of this device.

\section{REFERENCES}

American Heart Association (1974) Standards for cardiopulmonary resuscitation and emergency cardiac care fournal of the American Medical Association 227, 833-68. 
Elling R. \& Politis J. (1983) An evaluation of emergency medical technicians' ability to use manual ventilation devices. Annals of Emergency Medicine 12, 765-8.

Harrison R. R., Maull K. I., Keenan R. L. \& Boyan C. P. (1982) Mouth-to-mask ventilation: a superior method of rescue breathing. Annals of Emergency Medicine 11, 74-6.

Jesudian M. C., Harrison R. R., Keenan R. L. \& Maull K. I. (in press) Bag-valve-mask ventilation: two rescuers are better than one. Critical Care Medicine.

\section{K. I. MAULL}

Department of Surgery, University of Tennessee Memorial Research Center and Hospital, Knoxsville, Tennessee 